\title{
Magnetoelectric effect and the upper critical field in superconductors without inversion center
}

\author{
V.P. Mineev \\ Commissariat a l'Energie Atomique, INAC/SPSMS, Grenoble 38054, France \\ E-mail: vpmineev@yahoo.fr \\ Received February 25, 2011
}

\begin{abstract}
Solving the Ginzburg-Landau equation we demonstrate that the paramagnetic limiting of superconducting state in the superconductors without space inversion symmetry is significantly weakened. It is caused by so called magnetoelectric mechanism proving to be effective or not depending of the magnetic field direction and the crystal symmetry.
\end{abstract}

PACS: 74.25. $-\mathbf{q}$ Properties of superconductors;

75.85.+t Magnetoelectric effects, multiferroics.

Keywords: superconducting state, magnetoelectric mechanism, crystal symmetry.

\section{Introduction}

The term "magnetoelectric effect" in noncentrosymmetric superconductors encompasses several intriguing features. It has been discussed on a phenomenological level by introducing additional linear gradients terms to the Ginzburg-Landau (GL) free energy, so-called Lifshitz invariants, like

$$
\eta^{*}(\mathbf{r}) K_{i j} H_{i} D_{j} \eta(\mathbf{r})
$$

Here $\eta(\mathbf{r})$ denotes the order parameter of superconductor, $\mathbf{H}$ is magnetic field and $\mathbf{D}=-i \nabla-2 e \mathbf{A}$ is the gaugeinvariant gradient. First predicted by Levitov, Nazarov, and Eliashberg [1], the magnetoelectric effect was studied microscopically by several authors [2-6]. In this context several observable effects have been predicted: (i) the existence of a helically twisted superconducting order parameter in a magnetic field in two and three dimensional cases and spontaneous supercurrents in a 2D geometry [4-9] and near the superconductor surface [10] as well as along junctions of two superconductors with opposite directions of polarization [11], (ii) the augmentation of the upper critical field oriented perpendicular to the direction of the space parity breaking [6,8], (iii) magnetic interference patterns of the Josephson critical current for a magnetic field applied perpendicular to the junction [8].

The presence of Lifshitz invariants (1), however, can mislead to invalid conclusions like the inevitable appearance of modulated in space superconducting states or noticeable augmentation of the upper critical field $[6,8]$. Here we would like to give insight into this subtleties by dis- cussing the magnetic field dependence of the effective critical temperature in the Ginzburg-Landau framework. Considering paramagnetic limiting mechanism of superconductivity that was ignored in cited above studies we show that magnetoelectric effect weakens in many cases but never suppresses completely the paramagnetic limiting of the superconducting state. There is also pointed out that the order parameter helical modulation claimed in several papers presents nothing else as known Landau-Abrikosov degenerate solution of the Ginzburg-Landau equation. We begin with the short description of the electronic states in noncentrosymmetric metals.

\section{Electronic states in non-centrosymmetric metals}

Our starting point is the following Hamiltonian of noninteracting electrons in a crystal without inversion center:

$$
H_{0}=\sum_{\mathbf{k} \alpha \beta=\uparrow, \downarrow}\left[\xi(\mathbf{k}) \delta_{\alpha \beta}+\gamma(\mathbf{k}) \cdot \sigma_{\alpha \beta}\right] a_{\mathbf{k} \alpha}^{\dagger} a_{\mathbf{k} \beta}
$$

where $a_{\mathbf{k} \alpha}^{\dagger}\left(a_{\mathbf{k} \alpha}\right)$ creates (annihilates) an electronic state $|\mathbf{k} \alpha\rangle$. Furthermore, $\xi(\mathbf{k})=\varepsilon(\mathbf{k})-\mu$ denotes the spinindependent part of the spectrum measured relative to the chemical potential $\mu, \alpha, \beta=\uparrow, \downarrow$ are spin indices and $\sigma$ are the Pauli matrices. The sum over $\mathbf{k}$ is restricted to the first Brillouin zone. The second term in Eq. (2) describes the antisymmetric spin-orbit (SO) coupling whose form depends on the specific noncentrosymmetric crystal structure [12,13]. The pseudovector $\gamma(\mathbf{k})$ satisfies $\gamma(-\mathbf{k})=-\gamma(\mathbf{k})$ and $g \gamma\left(g^{-1} \mathbf{k}\right)=\gamma(\mathbf{k})$, where $g$ is any symmetry operation in the generating point group $\mathcal{G}$ of 
the crystal (see below). The usual symmetric spin-orbit coupling which is present also in centrosymmetric crystals yields a new spinor basis (pseudospinor) $\alpha, \beta$ in Eq. (2), which retains the ordinary spin-1/2 structure with complete $\mathrm{SU}(2)$-symmetry. This is different for the antisymmetric spin-orbit coupling. The effect of the antisymmetric spinorbit coupling is a spin splitting of the band energy with kdependent spin quantization axis which removes the SU(2)-symmetry.

Depending on the purpose it is more convenient to express the Hamiltonian (2) in the initial $2 \times 2$ matrix form (spinor representation) or in its diagonal form (band representation). The energy bands are given by

$$
\xi_{ \pm}(\mathbf{k})=\xi(\mathbf{k}) \pm|\gamma(\mathbf{k})|
$$

with the Hamiltonian

$$
H_{0}=\sum_{\mathbf{k}} \sum_{\lambda= \pm} \xi_{\lambda}(\mathbf{k}) c_{\mathbf{k} \lambda}^{\dagger} c_{\mathbf{k} \lambda}
$$

where the two sets of electronic operators are connected by a unitary transformation,

$$
a_{\mathbf{k} \alpha}=\sum_{\lambda} u_{\alpha \lambda}(\mathbf{k}) c_{\mathbf{k} \lambda}
$$

with

$$
\left(u_{\uparrow \lambda}(\mathbf{k}), u_{\downarrow \lambda}(\mathbf{k})\right)=\frac{\left(|\gamma|+\lambda \gamma_{z}, \lambda\left(\gamma_{x}+i \gamma_{y}\right)\right)}{\sqrt{2|\gamma|\left(|\gamma|+\lambda \gamma_{z}\right)}} .
$$

The Fermi surfaces defined by the equations $\xi_{ \pm}(\mathbf{k})=0$ are split, except at specific points or lines where $|\gamma(\mathbf{k})|=0$ is satisfied. The band dispersion functions $\xi_{\lambda}(\mathbf{k})$ are invariant with respect to all operations of $\mathcal{G}$ and the time reversal operations $K=i \hat{\sigma}_{2} K_{0}$ ( $K_{0}$ is the complex conjugation). The states $|\mathbf{k}, \lambda\rangle$ and $K|\mathbf{k}, \lambda\rangle$ belonging to the band energies $\xi_{\lambda}(\mathbf{k})$ and $\xi_{\lambda}(-\mathbf{k})$, respectively, are degenerate, since the time reversal operation yields $K|\mathbf{k}, \lambda\rangle=$ $=t_{\lambda}(\mathbf{k})|-\mathbf{k}, \lambda\rangle$, where $t_{\lambda}(\mathbf{k})=-t_{\lambda}(-\mathbf{k})$ is a nontrivial phase factor $[14,15]$. For the eigenstates of $H_{0}$, defined by (6), this phase factor takes the form

$$
t_{\lambda}(\mathbf{k})=-\lambda \frac{\gamma_{x}(\mathbf{k})-i \gamma_{y}(\mathbf{k})}{\sqrt{\gamma_{x}^{2}(\mathbf{k})+\gamma_{y}^{2}(\mathbf{k})}}
$$

Finally we turn to the basic form of the antisymmetric spin-orbit coupling as it results from the noncentrosymmetric crystal structures. Here we ignore the Brillouin zone structure and use only the expansion for small momenta $\mathbf{k}$ leading to basis functions satisfying the basic symmetry requirements of $\gamma(\mathbf{k})$. For the cubic group $\mathcal{G}=\mathbf{O}$, the point group of $\mathrm{Li}_{2}\left(\mathrm{Pd}_{1-x}, \mathrm{Pt}_{x}\right)_{3} \mathrm{~B}$ [16], the simplest form compatible with symmetry requirements is

$$
\gamma(\mathbf{k})=\gamma_{0} \mathbf{k}
$$

where $\gamma_{0}$ is a constant. For point groups containing improper elements, i.e., reflections and rotation-reflections, expressions become more complicated. The full tetrahedral group $\mathcal{G}=\mathbf{T}_{d}$, which is relevant for $\mathrm{Y}_{2} \mathrm{C}_{3}$ [17] and possibly $\mathrm{KOs}_{2} \mathrm{O}_{6}$, the expansion of $\gamma(\mathbf{k})$ starts with third order in the momentum,

$$
\gamma(\mathbf{k})=\gamma_{0}\left[k_{x}\left(k_{y}^{2}-k_{z}^{2}\right) \hat{x}+k_{y}\left(k_{z}^{2}-k_{x}^{2}\right) \hat{y}+k_{z}\left(k_{x}^{2}-k_{y}^{2}\right) \hat{z}\right] .
$$

This is sometimes called Dresselhaus spin-orbit coupling [12], and was originally discussed for bulk semiconductors of zinc-blend structure.

The tetragonal point group $\mathcal{G}=\mathbf{C}_{4 v}$, relevant for $\mathrm{CePt}_{3} \mathrm{Si}$ [18], $\mathrm{CeRhSi}_{3}$ [19] and $\mathrm{CeIrSi}_{3}$ [20], yields the antisymmetric spin-orbit coupling

$$
\gamma(\mathbf{k})=\gamma_{\perp}\left(k_{y} \hat{x}-k_{x} \hat{y}\right)+\gamma_{\|} k_{x} k_{y} k_{z}\left(k_{x}^{2}-k_{y}^{2}\right) \hat{z} .
$$

In the purely two-dimensional case, setting $\gamma_{\|}=0$ one recovers the Rashba interaction [13] which is often used to describe the effects of the absence of mirror symmetry in semiconductor quantum wells.

The single-electron Hamiltonian (2) can be extended to include the magnetic field as follows:

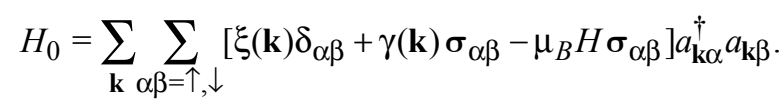

The last term describes the Zeeman interaction for an external magnetic field $\mathbf{H}$, with $\mu_{B}$ being the Bohr magneton. The orbital effect of the field can be included by replacing $\mathbf{k} \rightarrow \mathbf{k}+(e / \hbar c) \mathbf{A}(\hat{\mathbf{r}})$ [21], where $\hat{\mathbf{r}}=i \nabla_{\mathbf{k}}$ is the position operator in the $\mathbf{k}$-representation.

The eigenvalues of the Hamiltonian (11) are

$$
\xi_{\lambda}(\mathbf{k}, \mathbf{H})=\xi(\mathbf{k})+\lambda\left|\gamma(\mathbf{k})-\mu_{B} \mathbf{H}\right|
$$

There are two Fermi surfaces determined by the equations

$$
\xi_{\lambda}(\mathbf{k}, \mathbf{H})=0 .
$$

For certain directions and magnitudes of $\mathbf{H}$ there may be accidental degeneracies of the Fermi surfaces, determined by the equation $\gamma(\mathbf{k})=\mu_{B} \mathbf{H}$. However, there are no symmetry reasons for such intersections.

An important property of the Fermi surfaces (13) is the fact that their shapes depend on the magnetic field in a characteristic way, which can be directly probed by dHvA experiments $[22,23]$. Note that while at $H=0$ time reversal symmetry guarantees $\xi_{\lambda}(-\mathbf{k})=\xi_{\lambda}(\mathbf{k})$, the loss of time reversal symmetry for $H \neq 0$ yields, in general, $\xi_{\lambda}(-\mathbf{k}, \mathbf{H}) \neq \xi_{\lambda}(\mathbf{k}, \mathbf{H})$, i.e., the Fermi surfaces do not have inversion symmetry.

\section{Ginzburg-Landau theory for one band noncentrosymmetric superconductors}

Instead considering intrinsic multi-band situation due to the spin splitting of the electron band, for simplicity we restrict ourselves to a one-band situation, i.e., we ignore one of the two bands. This band shall be characterized by an isotropic density of states at the Fermi energy, $N_{0+}(\mathbf{k})=N_{+}$. 
The Ginzburg-Landau free energy functional in an uniaxial non-centrosymmetric superconductor one band superconductor is [6]

$$
\begin{aligned}
& F=\int d^{3} r\left\{\alpha\left(T-T_{c 0}\right)|\eta|^{2}+\right. \\
& \left.+\eta^{*}\left[K_{1}\left(D_{x}^{2}+D_{y}^{2}\right)+K_{2} D_{z}^{2}+K_{i j} H_{i} D_{j}+Q_{i j} H_{i} H_{j}\right] \eta\right\},
\end{aligned}
$$

where $\alpha=N_{0+} / 2 T_{c 0}$, and other coefficients are expressed through the averages over the Fermi surface of the products of components of the Fermi velocity and unit vector $\hat{\gamma}(\mathbf{k})$ :

$$
\begin{gathered}
K_{1}=\frac{N_{0+} S_{3}}{8}\left\langle v_{x}^{2}(\mathbf{k})\right\rangle, \quad K_{2}=\frac{N_{0+} S_{3}}{8}\left\langle v_{z}^{2}(\mathbf{k})\right\rangle, \\
K_{i j}=-\frac{\mu_{B} N_{0+} S_{3}}{2}\left\langle\hat{\gamma}_{i}(\mathbf{k}) v_{j}(\mathbf{k})\right\rangle, \\
Q_{i j}=\frac{\mu_{B}^{2} N_{0+} S_{3}}{2}\left\langle\hat{\gamma}_{i}(\mathbf{k}) \hat{\gamma}_{j}(\mathbf{k})\right\rangle, \\
S_{3}(T)=\pi T \sum_{n} \frac{1}{\left|\omega_{n}\right|^{3}}=\frac{7 \zeta(3)}{4 \pi^{2} T^{2}} .
\end{gathered}
$$

The term linear in $\mathbf{H}$ incorporates the magnetoelectric effects while the term quadratic in $\mathbf{H}$ describes the paramagnetic effect. Therefore $Q_{i j}|\eta|^{2}$ is connected with the change of the paramagnetic susceptibility in the superconducting phase compared with the normal state (Pauli) susceptibility. In particular, $Q_{i j}$ vanishes when there is no change of the paramagnetic susceptibility. These coefficients have to be compared with those of a spin singlet state of a centrosymmetric superconductor, $Q_{i j}^{(0)}=$ $=\delta_{i j} \mu_{B}^{2} N_{0} S_{3} / 2$ which, assuming $N_{0+}=N_{0}$, is larger than above $Q_{i j}$.

We consider now the two illustrative cases, the point group $C_{4 v}$ and $D_{4}$ which are characterized by the pseudovectors

$$
\begin{array}{ll}
\gamma(\mathbf{k})=\gamma_{\perp}\left(k_{y} \hat{x}-k_{x} \hat{y}\right)+\gamma_{\|} k_{x} k_{y} k_{z}\left(k_{x}^{2}-k_{y}^{2}\right) \hat{z}, & \text { for } C_{4 v}, \\
\gamma(\mathbf{k})=\gamma_{\perp}\left(k_{x} \hat{x}+k_{y} \hat{y}\right)+\gamma_{\|} k_{z} \hat{z}, & \text { for } D_{4} .
\end{array}
$$

For symmetry arguments and using above expressions we find the following relations for the coefficients,

$$
\begin{array}{llll}
K_{x y}=-K_{y x} \neq 0 & \text { and } & K_{i j}=0 & \text { otherwise, } \\
Q_{x x}=Q_{y y} \neq Q_{z z}>0 & \text { and } & Q_{i j}=0 & \text { otherwise }
\end{array}
$$

for $C_{4 v}$ where presumably $\left|K_{z z}\right| \ll\left|K_{x y}\right|$ and $Q_{z z} \ll Q_{x x}$ due to large number of nodes in the k-dependence of the $\gamma_{\|}$-part of $\gamma(k)$, and

$$
\begin{array}{ll}
K_{x x}=K_{y y} \neq 0, K_{z z} \neq 0 & \text { and } K_{i j}=0 \text { otherwise }, \\
Q_{x x}=Q_{y y} \neq Q_{z z}>0 & \text { and } Q_{i j}=0 \text { otherwise }
\end{array}
$$

for $D_{4}$.

\subsection{Symmetry $C_{4 v}, \mathbf{H} \| \hat{z}$}

In the case of $C_{4 v}$ for the field directed parallel to $z$ axis $\mathbf{H}=H(0,0,1)$ the terms linear in gradients and $\mathbf{H}$ is absent. The standard solution $\eta=\mathrm{e}^{i q} y^{y} f(x)$ of the GL equation

$$
\left\{\alpha\left(T-T_{c 0}\right)+K_{1}\left[-\frac{\partial^{2}}{\partial x^{2}}+\left(-i \frac{\partial}{\partial y}+2 e H x\right)^{2}\right]+Q_{z z} H^{2}\right\} \eta=0
$$

is degenerate in respect to $q_{y}$. The magnetic field dependence of critical temperature is

$$
T_{c}=T_{c 0}-\frac{2 e K_{1}}{\alpha} H-\frac{Q_{z z}}{\alpha} H^{2}
$$

Both the orbital (linear in $H$ ) and paramagnetic (quadratic in $H$ ) depairing effect are present. Compared to the ordinary spin-singlet case, however, the effect of the paramagnetic limiting is weaker here due to $Q_{z z}<Q_{z z}^{(0)}$. It is important to note here that no magnetoelectric effect comes into play here.

\subsection{Symmetry $D_{4}, \mathbf{H} \| \hat{z}$}

The situation is quite different for the uniaxial crystals with point symmetry group $D_{4}$ (or $D_{6}$ ). The GL equation includes gradient terms in the field direction and acquires the form

$$
\begin{aligned}
& \left\{\alpha\left(T-T_{c 0}\right)+K_{1}\left[-\frac{\partial^{2}}{\partial x^{2}}+\left(-i \frac{\partial}{\partial y}+2 e H x\right)^{2}\right]-\right. \\
& \left.-i K_{z z} H \frac{\partial}{\partial z}-K_{2} \frac{\partial^{2}}{\partial z^{2}}+Q_{z z} H^{2}\right\} \eta=0 .
\end{aligned}
$$

The solution can be written as

$$
\eta=\mathrm{e}^{i q} y^{y} \mathrm{e}^{i z_{z}^{z}} f(x)
$$

which remains degenerate with respect to the wavevector $q_{y}$, but not with respect to $q_{z}$ which is used to maximize the critical temperature to

$$
T_{c}=T_{c 0}-\frac{2 e K_{1}}{\alpha} H+\left(\frac{K_{z z}^{2}}{4 K_{2}}-Q_{z z}\right) \frac{H^{2}}{\alpha} .
$$

This corresponds to the finite wavevector

$$
q_{z}=\frac{K_{z z} H}{2 K_{2}}
$$

Note that this wave vector could also be absorbed into the vector potential without changing the physically relevant results: $\mathbf{A} \rightarrow \mathbf{A}+\nabla \chi$ with $\chi=-q_{z} z / 2 e$.

The simple paramagnetic depairing effect is weakened due to magnetoelectric response of the system. Adjusting the nucleation of the superconducting phase to the shifted 
Fermi surface, as incorporated in the wavevector $q_{z}$, recovers some of the strength of the nucleating condensate. This is a specific effect of the noncentrosymmetric superconductor and has its conceptional analogue in the FFLO phase for centrosymmetric spin singlet superconductors, where the condensate also nucleates with finite momentum Cooper pairs in order to optimize the pairing of degenerate quasiparticles on the split Fermi surface.

\subsection{Symmetry $C_{4 v}, \mathbf{H} \perp \hat{z}$}

Now we turn the magnetic field into the basal plane $\mathbf{H}=H(\cos \varphi, \sin \varphi, 0)$, and impose a gauge to have the vector potential $\mathbf{A}=H z(\sin \varphi,-\cos \varphi, 0)$. The corresponding GL equation take the form

$$
\begin{aligned}
& \left\{\alpha\left(T-T_{c 0}\right)+K_{1}\left(D_{x}^{2}+D_{y}^{2}\right)-K_{2} \frac{\partial^{2}}{\partial z^{2}}+\right. \\
& \left.+K_{x y}\left(H_{x} D_{y}-H_{y} D_{x}\right)+Q_{x x} H^{2}\right\} \eta=0,
\end{aligned}
$$

where

$$
D_{x}=-i \frac{\partial}{\partial x}+2 e H_{y} z, \quad D_{y}=-i \frac{\partial}{\partial y}-2 e H_{x} z
$$

Like in ordinary superconductors the solution of this equation have the Abrikosov form

$$
\eta(\mathbf{r})=\exp \left[i(\mathbf{p} \times \mathbf{r})_{z}\right] f(z),
$$

where we write $\mathbf{p}=p \mathbf{H} / H$ as a vector parallel to the magnetic field $\left((\mathbf{p} \times \mathbf{r})_{z}\right.$ denoting the $z$-component of the vector $\mathbf{p} \times \mathbf{r})$, and $f(z)$ satisfies the resulting renormalized harmonic oscillator equation

$$
\begin{aligned}
& \left\{\alpha\left(T-T_{c 0}\right)+K_{1}(2 e H)^{2}\left(z-z_{0}\right)^{2}-\right. \\
& \left.-K_{2} \frac{\partial^{2}}{\partial z^{2}}+\left(Q_{x x}-\frac{K_{x y}^{2}}{4 K_{1}}\right) H^{2}\right\} f(z)=0,
\end{aligned}
$$

with the shifted equilibrium position

$$
z_{0}=(2 e H)^{-1}\left(p+\frac{K_{x y}}{2 K_{1}} H\right)
$$

Thus, the vector $\mathbf{p}$ is absorbed into the shift $z_{0}$ and does not appear anywhere else in the equation.

Then the corresponding eigenvalue determines the magnetic field dependence of optimized critical temperature:

$$
T_{c}=T_{c 0}-\frac{2 e \sqrt{K_{1} K_{2}}}{\alpha} H+\left(\frac{K_{x y}^{2}}{4 K_{1}}-Q_{x x}\right) \frac{H^{2}}{\alpha} .
$$

The eigenstates are degenerate with respect to $p$ and acquire the same structure as in the case of the usual Landau degeneracy following from the system translational invariance in the plane perpendicular to magnetic field. Nevertheless, the characteristics of the noncentrosymmetricity incorporated in the $K_{i j}$-terms appears in the expression of $T_{c}$. Similar to the previous case of $D_{4}$ with $\mathbf{H} \| z$ the magnetoelectric effect yields a reduction of the paramagnetic limiting term. This renormalization is surprisingly strong in general, as we can see when we return to the expressions which we had derived for the different coefficients. We obtain for the last term in Eq. (32),

$$
\left[\frac{K_{x y}^{2}}{4 K_{1}}-Q_{x x}\right] \frac{H^{2}}{\alpha}=\left[\frac{\left\langle\gamma_{x}(\mathbf{k}) v_{y}(\mathbf{k})\right\rangle^{2}}{\left\langle v_{x}^{2}(\mathbf{k})\right\rangle}-\left\langle\hat{\gamma}_{x}^{2}(\mathbf{k})\right\rangle\right] \frac{\mu_{B}^{2} H^{2} N_{+} S_{3}}{2 \alpha} .
$$

Considering the simplified picture of a parabolic band with $\mathbf{v}(\mathbf{k})=\mathbf{k} / m^{*}$ and a Rashba spin-orbit coupling $\hat{\gamma}=\mathbf{k} \times \hat{z}$ (setting $\gamma_{z}(\mathbf{k})=0$ ) we find the amazing result that the two terms exactly cancel and the paramagnetic effect is completely suppressed. This effect can be immediately obtained, if we perform the gauge transformation

$$
\mathbf{A} \rightarrow \mathbf{A}+\frac{\mu_{B} m^{*}(\hat{z} \times \mathbf{H})}{e k_{F}}
$$

eliminating the paramagnetic term in initial equation. However, it is important to notice that this exact cancellation is a consequence of the simplified forms of the band structure and the spin-orbit coupling term. Taking more realistic band structure effects into account it is obvious that this identity does not hold anymore in general. Nevertheless, our results suggests that the magnetoelectric effect can, in principle, yield a substantial contribution to eliminate the paramagnetic limiting also for fields in the basal plane.

\subsection{Symmetry $D_{4}, \mathbf{H} \perp \hat{z}$}

It is easy to see that this case is analogue to the situation for the field along the $z$-axis and has only quantitative differences. Thus also here we encounter a reduction of the paramagnetic limit due to the magnetoelectric effect yielding

$$
T_{c}=T_{c 0}-\frac{2 e \sqrt{K_{1} K_{2}}}{\alpha} H+\left(\frac{K_{x x}^{2}}{3 K_{1}}-Q_{x x}\right) \frac{H^{2}}{\alpha},
$$

where also the same considerations concerning the gauge freedom apply as in the case of $\mathbf{H} \| \hat{z}$ apply.

\subsection{Two-dimensional case, symmetry $C_{4 v}, \mathbf{H} \perp \hat{z}$}

The simplest way to pass from 3D to $2 \mathrm{D}$ situation it is to introduce $\delta(z)$ function potential well into 3D GL equation (27). It is equivalent to the theory used by Tinkham [24] for the calculation of the upper critical field in a thin film with thickness $d<<\xi$ for a field parallel to the film. Thus, we consider the equation 


$$
\begin{aligned}
& \left\{\alpha\left(T-T_{c 0}\right)-K_{1}\left(D_{x}^{2}+D_{y}^{2}\right)-K_{2} \frac{\partial^{2}}{\partial z^{2}}+\right. \\
& \left.+K_{x y}\left(H_{x} D_{y}-H_{y} D_{x}\right)+Q_{x x} H^{2}-\frac{2 K_{2}}{d} \delta(z)\right\} \eta=0,
\end{aligned}
$$

where $d$ is a length of the order of the film thickness that is in pure $2 \mathrm{D}$ case it is an atomic scale length. This eigenvalue equation has the solution

$$
\eta(\mathbf{r})=A \exp \left[i(\mathbf{p} \times \mathbf{r})_{z}\right] \exp \left(-\frac{|z|}{d}\right),
$$

where $\mathbf{p}=p \mathbf{H} / H$ is a vector with arbitrary length directed along magnetic field. This then determines the critical temperature as a function of the applied magnetic field:

$$
\alpha\left(T-\tilde{T}_{c 0}\right)+K_{1}(2 e H)^{2}\left\langle\left(z-z_{0}\right)^{2}\right\rangle+\left(Q_{x x}-\frac{K_{x y}^{2}}{4 K_{1}}\right) H^{2}=0 .
$$

Here $\tilde{T}_{c 0}$ is the critical temperature in the absence of a magnetic field, corresponding to $d^{2}=K_{2} / \alpha\left(\tilde{T}_{c 0}-T_{c 0}\right)$. Moreover, $\langle\ldots\rangle$ denotes the expectation value using the wave function $\exp (-|z| / d)$ and $z_{0}$ is determined by the same expression as in the $3 \mathrm{D}$ case

$$
z_{0}=(2 e H)^{-1}\left(p+\frac{K_{x y}}{2 K_{1}} H\right)
$$

Hence, we obtain for the critical temperature

$$
T_{c}=\tilde{T}_{c 0}+\left[\frac{K_{x y}^{2}}{4 K_{1}}-Q_{x x}\right] \frac{H^{2}}{\alpha}-\frac{K_{1}}{\alpha}\left(z_{0}^{2}+d^{2} / 2\right)(2 e H)^{2} .
$$

The critical temperature reaches obviously a maximal value at $z_{0}=0$, i.e., for

$$
p=-\frac{K_{x y}}{2 K_{1}} H .
$$

Thus, unlike to the $3 \mathrm{D}$ geometry the $2 \mathrm{D}$ superconducting state proves to be modulated in the perpendicular to the field direction. The upper critical field shows also here the square root temperature dependence usual for thin films in a parallel magnetic field [24]. Under special conditions (e.g., rotation symmetry around the normal vector of the film) the expression in the square parenthesis in Eq. (40) may vanish, as described above. Then, unlike in usual superconductors, non-centrosymmetric superconductors follow the standard Tinkham behavior unchanged by paramagnetic contributions.

In view of strong inequality $d \ll 1 / \sqrt{2 e H}$ the complete suppression of 2D superconducting state $\left(T_{c}(H)=0\right)$ is reached in the field which exceeds the orbital critical field in the $3 \mathrm{D}$ case (32).

\section{Conclusion}

One of the physically most remarkable aspects of noncentrosymmetric superconductivity is connected with magnetoelectricity, the peculiar connection between supercurrents and spin polarization. We have considered one aspect in this context, namely its influence on paramagnetic limiting. This effect is of interest in strongly correlated electron systems where the coherence length is generally small due to the enhanced masses like in heavy Fermion compounds. Here ordinary orbital depairing in a magnetic field is weak, such that the upper critical field reaches magnitudes where paramagnetic limiting through spin polarization becomes visible. There was demonstrated that depending of crystal symmetry and field direction the paramagnetic suppression of superconducting state is substantially weakened by the magnetoelectric effect. In some cases the latter causes the appearence FFLO like states for centrosymmetric superconductors. In other the modulated state does not appear obeying just usual property of the Landau-Abrikosov degeneracy of the Ginzburg-Landau equation. The conclusions derived here in frame of one band superconductivity model allow generalization to the more real two-band case.

Author express the gratitude to V.G. Peschanskii for the numerous kind conversations.

1. L.S. Levitov, Yu.V. Nazarov, and G.M. Eliashberg, Pis'ma Zh. Eksp. Teor. Fiz. 41, 365 (1985) [JETP Lett. 41, 445 (1985)].

2. V.M. Edel'stein, Zh. Eksp. Teor. Fiz. 95, 2151 (1989) [Sov. Phys. JETP 68, 1244 (1989)].

3. V.M. Edel'stein, Phys. Rev. Lett. 75, 2004 (1995).

4. V.M. Edel'stein, J. Phys.: Condens. Matter 8, 339 (1996).

5. S.K. Yip, Phys. Rev. B65, 144508 (2002).

6. K.V. Samokhin, Phys. Rev. B70, 104521 (2004).

7. O.V. Dimitrova and M.V. Feigel'man, Pis'ma ZhETF 78, 1132 (2003); Phys. Rev. B76, 014522 (2007).

8. R.P. Kaur, D.F. Agterberg, and M. Sigrist, Phys. Rev. Lett. 94, 137002 (2005).

9. D.F. Agterberg and R.P. Kaur, Phys. Rev. B75, 064511 (2007)

10. M. Oka, M. Ishioka, and K. Machida, Phys. Rev. B73, 214509 (2006).

11. S. Fujimoto, Phys. Rev. B72, 024515 (2005).

12. G. Dresselhaus, Phys. Rev. 100, 580 (1955); L.M. Roth, Phys. Rev. 173, 755 (1968).

13. E.I. Rashba, Fiz. Tverd. Tela (Leningrad) 2, 1224 (1960) [Sov. Phys. Solid State 2, 1109 (1960)].

14. L.P. Gor'kov and E.I. Rashba, Phys. Rev. Lett. 87, 037004 (2001).

15. I.A. Sergienko and S.H. Curnoe, Phys. Rev. B70, 214510 (2004).

16. K. Togano, P. Badica, Y. Nakamori, S. Orimo, H. Takeya, and K. Hirata, Phys. Rev. Lett. 93, 247004 (2004); P. Badica, 
T. Kondo, and K. Togano, J. Phys. Soc. Jpn. 74, 1014 (2005).

17. G. Amano, S. Akutagawa, T. Muranaka, Y. Zenitani, and J. Akimitsu, J. Phys. Soc. Jpn. 73, 530 (2004).

18. E. Bauer, G. Hilscher, H. Michor, Ch. Paul, E.W. Scheidt, A. Gribanov, Yu. Seropegin, H. Noël, M. Sigrist, and P. Rogl, Phys. Rev. Lett. 92, 027003 (2004).

19. N. Kimura, K. Ito, K. Saitoh, Y. Umeda, H. Aoki, and T. Terashima, Phys. Rev. Lett. 95, 247004 (2005).

20. I. Sugitani, Y. Okuda, H. Shishido, T. Yamada, A. Thamizhavel, E. Yamamoto, T.D. Matsuda, Y. Haga, T. Takeuchi,
R. Settai, and Y. Onuki, J. Phys. Soc. Jpn. 75, 043703 (2006).

21. E.M. Lifshitz and L.P. Pitaevskii, Statistical Physics, Butterworth-Heinemann, Oxford (1995), P. 2.

22. V.P. Mineev and K.V. Samokhin, Phys. Rev. B72, 212504 (2005).

23. T. Terashima, M. Kimata, S. Uji, T. SugaWara, N. Kimura, H. Aoki, and H. Harima, Phys. Rev. B78, 205107 (2008).

24. M. Tinkham, Introduction to Superconductivity, McGrawHill, New York (1996). 